\title{
Manipulation of polarization conversion and multiplexing via all-silicon phase-modulated metasurfaces
}

\author{
Zhen Yue (岳 震) ${ }^{1}$, Jitao Li (李继涛)' ${ }^{1}$, Chenglong Zheng (郑程龙) ${ }^{1}$, Jie Li (李 杰) ${ }^{1}$, Mingyang Chen (陈明阳) ${ }^{2}$, \\ Xuanruo Hao (郝璇若) ${ }^{1}$, Hang Xu (徐 航) ${ }^{1}$, Qi Wang (汪 奇) $)^{1}$, Yating Zhang (张雅婷 $)^{1^{*}}$, and Jianquan Yao (姚建铨) $)^{1^{* *}}$ \\ ${ }^{1}$ Key Laboratory of Opto-Electronics Information Technology (Tianjin University], Ministry of Education, School of Precision Instruments and Opto-Electronics \\ Engineering, Tianjin University, Tianjin 300072, China \\ ${ }^{2}$ Department of Optoelectronic Information Science and Engineering, Jiangsu University, Zhenjiang 212013, China
}

*Corresponding author: yating@tju.edu.cn

${ }^{*}$ Corresponding author: jqyao@tju.edu.cn

Received December 3, 2021 | Accepted January 18, 2022 | Posted Online February 15, 2022

\begin{abstract}
Phase-modulated metasurfaces that can implement the independent manipulation of co- and cross-polarized output waves under circularly polarized (CP) incidence have been proposed. With this, we introduce one particular metasurface composed of meta-atoms with a phase difference of $2 \pi / 3$ to generate specific elliptically polarized waves under various polarized incidences. Furthermore, a metasurface composed of these above meta-atoms and the meta-atoms with a phase difference of $\pi / 3$ arranged in a certain rule can realize polarization conversion function between linearly polarized and CP states. The designs shed new light on multifarious optical devices and may further promote the development of metasurface polarization optics.
\end{abstract}

Keywords: phase-modulated metasurfaces; polarization conversion; multiplexing.

DOI: 10.3788/COL202220.043601

\section{Introduction}

In recent years, artificially engineered metasurfaces, which can effectively manipulate the polarization, amplitude, and phase of electromagnetic waves on the two-dimensional (2D) scale, have attracted more and more attention ${ }^{[1-3]}$. Compared with conventional optical devices dependent on phase accumulation, metasurfaces can obtain abrupt phase change within subwavelength thickness by appropriately changing specific parameters, thereby generating various functions ${ }^{[4,5]}$. Specifically, metasurfaces can generate spatial phase discontinuities over the entire $2 \pi$ range of a specific frequency, which have manifested that they can be utilized in a flat wave plate ${ }^{[6,7]}$, hologram imaging ${ }^{[8-10]}$, beam deflectors ${ }^{[11,12]}$, achromatic focusing lenses $^{[13,14]}$, spin-orbital angular momentum manipulation ${ }^{[15-18]}$, and other fields.

Pancharatnam-Berry (PB) phase, also called geometric phase, has been proposed to achieve phase-wavefront manipulation of circularly polarized $(\mathrm{CP})$ waves ${ }^{[19,20]}$. However, the output waves of $\mathrm{PB}$ phase metasurfaces under orthogonal $\mathrm{CP}$ waves have inherent correlation. To break this restriction, spin-decoupled metasurfaces combining PB phase with dynamic phase were designed to independently manipulate the output waves under left-handed CP (LCP) and right-handed CP (RCP) incidences ${ }^{[12,18,21,22]}$. Spin-decoupled metasurfaces have many applications in holography ${ }^{[21]}$, focusing ${ }^{[18]}$, and polarization multiplexing ${ }^{[22]}$.

It is worth noting that the above-mentioned metasurfaces all have the properties of a half-wave plate, so the wavefront phase manipulation is only achieved for cross-polarized waves. Once the metasurface no longer has the properties of a half-wave plate, the outgoing wave will exist with LCP and RCP components under $\mathrm{CP}$ incidence, meaning that it makes sense to independently manipulate cross and co-polarization. Recently, phase-modulated metasurfaces, which can realize the independent manipulation of co- and cross-polarized output waves under CP incidence, have been reported ${ }^{[23-25]}$. In this paper, a metasurface composed of meta-atoms with a phase difference of $2 \pi / 3$ between linear orthogonal polarizations is introduced to generate LCP and RCP waves with different amplitudes under CP wave incidence so as to be superimposed into a specific elliptically polarized (EP) wave (see Fig. 1). As shown in Fig. 1(b), the metasurface composed of meta-atoms with a phase difference of $2 \pi / 3$ and a phase difference of $\pi / 3$ according to certain rules can generate focused linearly polarized (LP) (CP) exit waves under CP (LP) incidence. The demonstration of specific polarization conversion and multiplexing achieved at the all-silicon metasurfaces opens new ways to polarize metasurfaces with full potentials and can be hopefully further extended to other frequency regimes. 


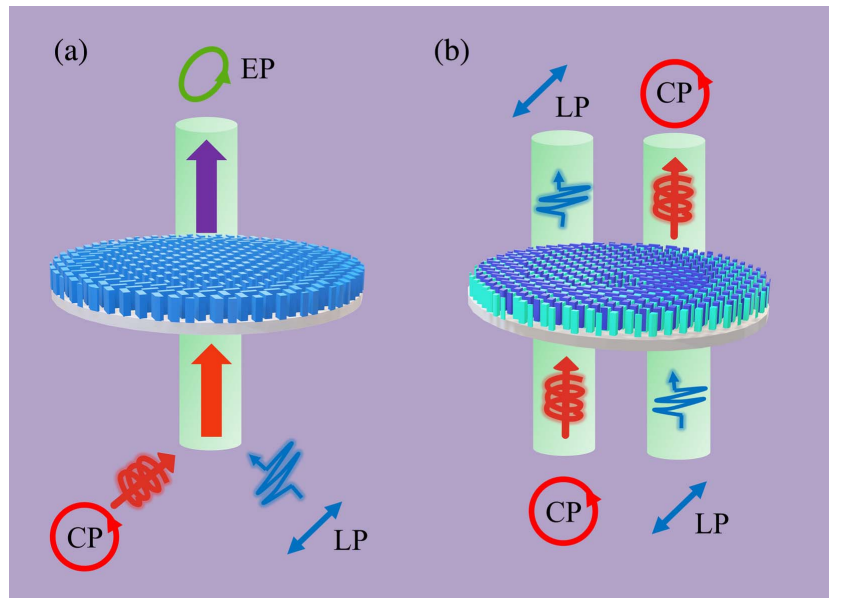

Fig. 1. Polarization conversion under different polarized incidences. (a) Schematic of a metasurface with polarized conversion, which converts the LP waves or CP waves into specific EP waves and focuses on one point, simultaneously. (b) Schematic of the proposed polarizer that can realize the function of mutual polarization conversion between linear polarization and circular polarization by a design based on an all-silicon metasurface.

\section{Results and Discussion}

\subsection{One EP wave generator}

Here, we demonstrate the feasibility of the concept through the derivation of the Jones matrix:

$$
J(\theta)=\left[\begin{array}{cc}
\cos \theta & -\sin \theta \\
\sin \theta & \cos \theta
\end{array}\right]\left[\begin{array}{cc}
\left|t_{x x}\right| e^{i \varphi_{x x}} & 0 \\
0 & \left|t_{y y}\right| e^{i \varphi_{y y}}
\end{array}\right]\left[\begin{array}{cc}
\cos \theta & \sin \theta \\
-\sin \theta & \cos \theta
\end{array}\right],
$$

where $\theta$ is the angle at which the meta-atom rotates counterclockwise around the $+z$ direction, which originates from the rotation matrix $\left[\begin{array}{cc}\cos \theta & \sin \theta \\ -\sin \theta & \cos \theta\end{array}\right], \varphi_{x x}, \varphi_{y y},\left|t_{x x}\right|$, and $\left|t_{y y}\right|$ represent corresponding phase delays and amplitudes along the $x$ and $y$ axis under $x$ and $y$ LP incidences. It is supposed that $\left|t_{x x}\right|=\left|t_{y y}\right|$ and the phase difference along $x$ and $y$ axes $\Delta \varphi$ is equal to $\varphi_{x x}-\varphi_{y y}$. Besides, the summation of propagation phase is expressed as $\sum \varphi=\varphi_{x x}+\varphi_{y y}$.

For input with the RCP state as $|\mathbf{R}\rangle=\left[\begin{array}{l}1 \\ i\end{array}\right]$, the exit field can be described as ${ }^{[25]}$

$$
\begin{aligned}
J(\theta) \cdot|\mathbf{R}\rangle= & \sin \left(\frac{1}{2} \Delta \varphi\right) \cdot e^{i\left(\frac{1}{2} \sum \varphi-\frac{\pi}{2}+2 \theta\right)}|\mathbf{L}\rangle \\
& +\cos \left(\frac{1}{2} \Delta \varphi\right) \cdot e^{i \frac{1}{2} \sum \varphi}|\mathbf{R}\rangle .
\end{aligned}
$$

Similarly, for the incident LCP wave $\left(|\mathbf{L}\rangle=\left[\begin{array}{c}1 \\ -i\end{array}\right]\right)$, the electric field of the transmitted wave generated is

$$
\begin{aligned}
J(\theta) \cdot|\mathbf{L}\rangle= & \sin \left(\frac{1}{2} \Delta \varphi\right) \cdot e^{i\left(\frac{1}{2} \sum \varphi-\frac{\pi}{2}-2 \theta\right)}|\mathbf{R}\rangle \\
& +\cos \left(\frac{1}{2} \Delta \varphi\right) \cdot e^{i \frac{i}{2} \sum \varphi}|\mathbf{L}\rangle .
\end{aligned}
$$

Surprisingly, Eqs. (2) and (3) indicate that the transmitted waves both exist with LCP and RCP components under LCP or RCP incidence. Moreover, under $\mathrm{CP}$ incidence, the amplitudes of the co- and cross-polarized components of the transmitted wave are $\cos (\Delta \varphi / 2)$ and $\sin (\Delta \varphi / 2)$, respectively.

Once it is guaranteed that the rotation angle $\theta$ is equal throughout to $\pi / 4$, then Eqs. (2) and (3) can be respectively simplified as

$$
\begin{aligned}
& J(\theta) \cdot|\mathbf{R}\rangle=\left[\sin \left(\frac{1}{2} \Delta \varphi\right) \cdot|\mathbf{L}\rangle+\cos \left(\frac{1}{2} \Delta \varphi\right) \cdot|\mathbf{R}\rangle\right] \cdot e^{i \frac{1}{2} \sum \varphi} \\
& J(\theta) \cdot|\mathbf{L}\rangle=\left[-\sin \left(\frac{1}{2} \Delta \varphi\right) \cdot|\mathbf{R}\rangle+\cos \left(\frac{1}{2} \Delta \varphi\right) \cdot|\mathbf{L}\rangle\right] \cdot e^{i \frac{1}{2} \sum \varphi}
\end{aligned}
$$

It can be readily seen from Eqs. (4) and (5) that by adjusting the phase difference $\Delta \varphi$, the desired EP exit wave can be obtained under the CP illumination.

In order to verify the feasibility of the above concept, we apply the anisotropic all-silicon meta-atoms A [permittivity $\varepsilon=11.9$, see Fig. 2(a)] with the phase difference $\Delta \varphi=2 \pi / 3$ as the basic elements for the metasurface construction. The thickness of the (a)

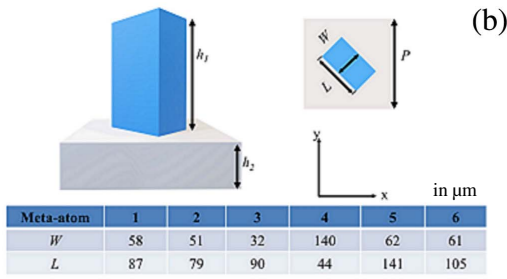

(c)

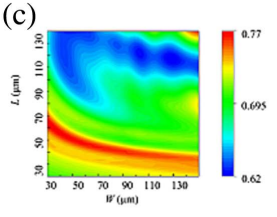

(f)

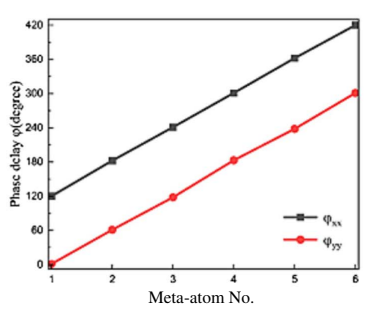

(b)

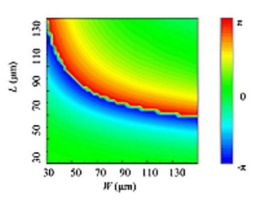

(d)

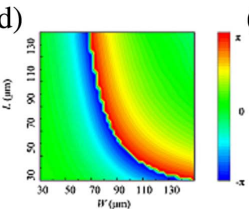

(e)

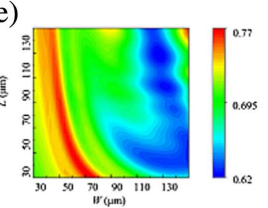

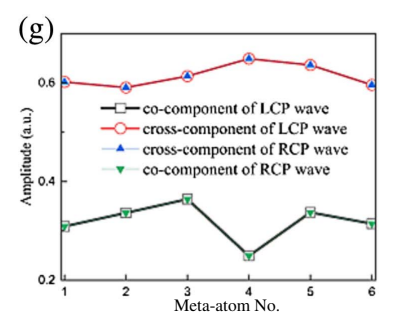

Fig. 2. (a) Schematic of the all-silicon pillars. (b)-(e) Schematic of the corresponding phase shifts and amplitudes under $x$-LP ( $y$-LP) incidence at $0.95 \mathrm{THz}$. (f) Simulated phase delays of the transmitted $x$ and $y$ components of six metaatoms $A$ in (a). (g) Simulated amplitudes under LCP (RCP) incidences. 
substrate is $h_{2}=300 \mu \mathrm{m}$ with a period of $P=150 \mu \mathrm{m}$. By rotating the silicon pillar with height $h_{1}=200 \mu \mathrm{m}$, width $W$, and length $L$, the rotation angle is $45^{\circ}$ to the $y$ axis, and then the final structure is formed. CST MICROWAVE STUDIO (2019) is exploited to simulate the transmission phase shifts and amplitudes of meta-atoms A. The simulated phase shift and amplitude of the exit wave under $x$-LP illumination at $0.95 \mathrm{THz}$ are shown in Figs. 2(b) and 2(c) (here, the angle between the rectangular silicon pillar and the $x$ axis is $0^{\circ}$ ). Figures 2(d) and 2(e) depict the corresponding phase shift and amplitude under $y$-LP incidence. It is worth noting that a phase variation of $2 \pi$ can be generated regardless of whether the incident wave is $x \mathrm{LP}$ or $y \mathrm{LP}$, which is crucial for metasurfaces design. After a quite careful selection, six types of meta-atoms A [see Fig. 2(a) for specific parameters] are utilized for metasurface design. Figure 2(f) presents phase delays $\varphi_{x x}$ and $\varphi_{y y}$ of transmission waves under $x$-LP or $y$-LP incidence; meanwhile, the corresponding amplitudes of the cross and co-components under $\mathrm{CP}$ incidence are shown in Fig. 2(g). Obviously, the phase variation of $2 \pi$ is well covered by meta-atoms A. It can be seen from Fig. 2(g) that the amplitudes of cross and co-polarizations of the meta-atoms $\mathrm{A}$ are consistent with the expected concepts, which can be exploited to construct a metasurface that can generate ellipsoidal polarization under LP or CP incidence. By this time, according to Eq. (4), it can be found that when the RCP wave is incident, the transmitted wave can be expressed as $\left[\begin{array}{c}\sqrt{3}+1 \\ (1-\sqrt{3}) i\end{array}\right]$. In addition, the exit EP wave can be described as $\left[\begin{array}{c}1-\sqrt{3} \\ -(\sqrt{3}+1) i\end{array}\right]$ under LCP incidence. After theoretical calculations, we can also conclude that the generated EP wave can be described as $\left[\begin{array}{c}1 \\ -\sqrt{3} i\end{array}\right]\left(\left[\begin{array}{c}\sqrt{3} \\ i\end{array}\right]\right)$ under $x$-LP $(y-\mathrm{LP})$ incidence.

Next, we design a metasurface composed of the meta-atoms A [see Fig. 2(a)] that meet a specific phase distribution, which can generate focused EP waves under LP or CP incidences, to demonstrate the hypothesis. To realize the beam focusing, the phase distribution of the meta-atoms A needs to meet the following conditions:

$$
\varphi=\frac{1}{2} \sum \varphi=-\frac{2 \pi}{\lambda}\left(\sqrt{f^{2}+r^{2}}-f\right),
$$

where $\lambda$ is the incident wavelength corresponding to $0.95 \mathrm{THz}$, $f=4.8 \mathrm{~mm}$ stands for focal length, and $r$ represents the distance from any point on the metasurface to the center of its circle.

The results of polarization conversion and focusing generated by the metasurface under the incidence of different polarized waves are obtained through simulation, as shown in Fig. 3. To more intuitively verify that the metasurface can generate specific EP waves under different polarized waves, the simulated results of its orthogonally polarized waves are also displayed here. It is noted that the intensity of the EP wave $\left(\left[\begin{array}{c}\sqrt{3}+1 \\ (1-\sqrt{3}) i\end{array}\right]\right)$ is concentrated in a certain place in space, while the intensity of its (a)

(b)

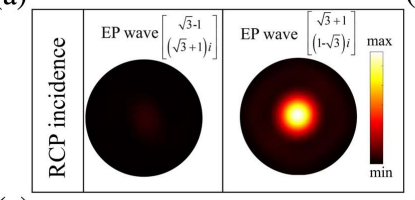

(c)

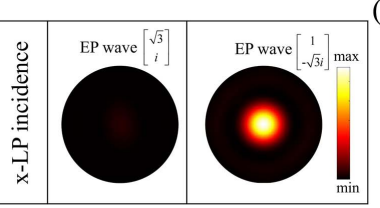

(d)
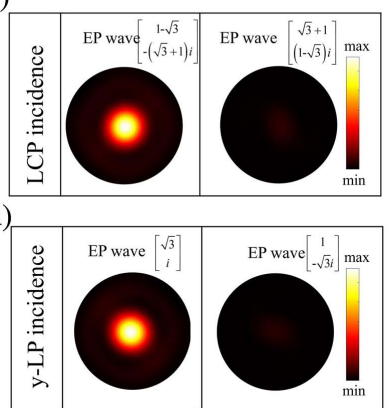

Fig. 3. Transmission characteristics of the proposed metasurface. (a)-(d) The intensity distributions of the desired EP wave and its orthogonally polarized wave on the focal plane under CP or LP illumination.

orthogonally polarized wave $\left(\left[\begin{array}{c}\sqrt{3}-1 \\ (\sqrt{3}+1) i\end{array}\right]\right)$ is negligible [see Fig. 3(a)]. Figures 2(b)-2(d) show the simulated results under LCP, $x$-LP, and $y$-LP incidences, which are consistent with the results obtained by the above calculations. Furthermore, comparing the simulation results of LCP and RCP incidence, it is not difficult to find that under the incidence of orthogonal CP waves, the EP waves generated by the metasurface are also orthogonal, similar to the case under the orthogonal LP incidences. In short, through setting a specific phase difference $\Delta \varphi$, the expected EP waves can be generated under the incidence of different polarized waves.

\subsection{Polarization multiplexed metasurface}

We consider introducing another kind of meta-atoms (called meta-atoms B) to form a new metasurface together with the meta-atoms $\mathrm{A}$, realizing the mutual polarization conversion between linear polarization and circular polarization. For RCP incidence, the meta-atoms $\mathrm{B}$ with the phase difference $\Delta \varphi_{1}=$ $\pi-\Delta \varphi$ will bring about an EP wave that can be described as

$$
\begin{aligned}
J(\theta) \cdot|\mathbf{R}\rangle & =\left[\sin \left(\frac{1}{2} \Delta \varphi_{1}\right) \cdot|\mathbf{L}\rangle+\cos \left(\frac{1}{2} \Delta \varphi_{1}\right) \cdot|\mathbf{R}\rangle\right] \cdot e^{\frac{i \frac{1}{2}}{2} \varphi_{1}} \\
& =\left[\cos \left(\frac{1}{2} \Delta \varphi\right) \cdot|\mathbf{L}\rangle+\sin \left(\frac{1}{2} \Delta \varphi\right) \cdot|\mathbf{R}\rangle\right] \cdot e^{i \frac{1}{2} \sum \varphi_{1}},
\end{aligned}
$$

where $\sum \varphi_{1}$ is the summation of propagation phase along the $x$ and $y$ directions. Once $\sum \varphi$ and $\sum \varphi_{1}$ in Eqs. (4) and (7) remain equal, the superposition of the two meta-atoms with phase differences of $\Delta \varphi$ and $\Delta \varphi_{1}$ can generate $x$-LP waves under RCP incidence. Besides, under LCP illumination, EP waves generated by the meta-atom with the phase difference $\Delta \varphi_{1}$ are

$J(\theta) \cdot|\mathbf{L}\rangle=\left[-\cos \left(\frac{1}{2} \Delta \varphi\right) \cdot|\mathbf{R}\rangle+\sin \left(\frac{1}{2} \Delta \varphi\right) \cdot|\mathbf{L}\rangle\right] \cdot e^{i \frac{1}{2} \sum \varphi_{1}}$. 
Obviously, the above scheme can also generate $y$-LP waves under the incidence of LCP waves. After further derivation, it can be found that this polarization multiplexing method can effectively achieve the polarization conversion between linear polarization and circular polarization.

With the proposed scheme, the six meta-atoms B with a phase difference of $\pi / 3$ are carefully selected, with parameters shown in Fig. 4. Comparing Fig. 2(f) with Fig. 4(a), it can be seen that the difference between the phase delay $\varphi_{x x}$ of the meta-atom A and the phase delay $\varphi_{x x}^{\prime}$ of the meta-atom B is $\pi / 6$. Substituting the phase difference $\Delta \varphi_{1}=\pi / 3$ into Eqs. (7) and (8), we can get the following conclusion: when RCP, LCP, $x$-LP and $y-\mathrm{LP}$ waves are incident on the meta-atom $\mathrm{B}$, the polarized waves generated are $\left[\begin{array}{c}\sqrt{3}+1 \\ (\sqrt{3}-1) i\end{array}\right],\left[\begin{array}{c}\sqrt{3}-1 \\ (-\sqrt{3}-1) i\end{array}\right],\left[\begin{array}{c}\sqrt{3} \\ -i\end{array}\right]$, and $\left[\begin{array}{c}1 \\ \sqrt{3} i\end{array}\right]$, respectively.

Then, we introduce the way of spatial interleaving to arrange the meta-atoms $\mathrm{A}$ and $\mathrm{B}$, so as to construct a metasurface with the function of polarization multiplexing. For the metaatoms $\mathrm{A}$, the phase distribution satisfies Eq. (6), and the frequency of incident wave and focal length are $0.95 \mathrm{THz}$ and $4.8 \mathrm{~mm}$, respectively. Similarly, considering a focused

(a)

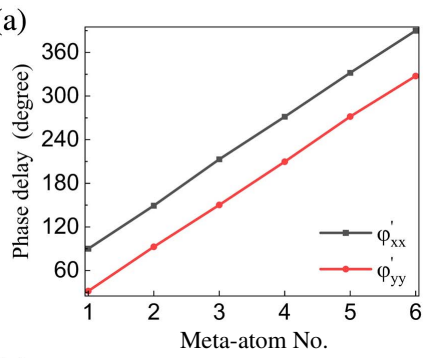

(c)

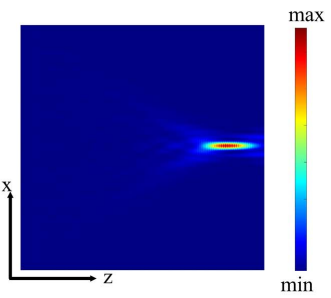

(b)

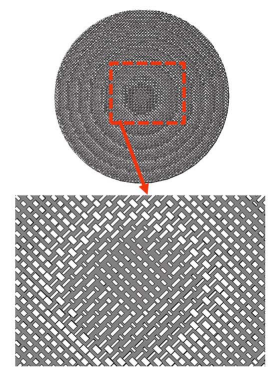

(d)

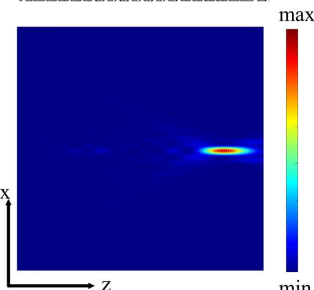

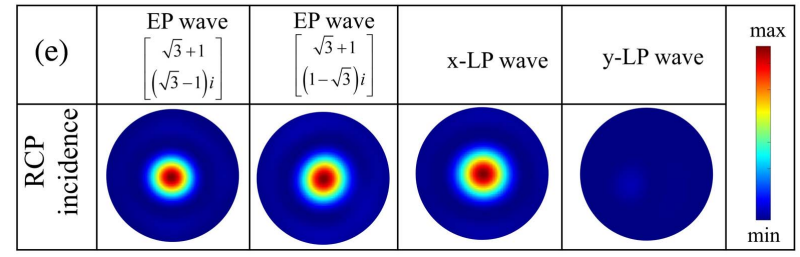

Fig. 4. (a) Simulated phase delays along the $x$ and $y$ components in the transmission direction. (b) Structure image of the polarization multiplexed metasurface. (c), (d) Simulated intensity profiles of EP waves generated by meta-atoms A and B on the xoz plane. (e) Simulated results of polarized waves on the focal plane $4.8 \mathrm{~mm}$ away from the metasurface under RCP incidence. polarized wave generated by meta-atoms B with a specific phase arrangement, the corresponding phase distribution is

$\varphi_{1}=\frac{1}{2} \sum \varphi_{1}=-\frac{2 \pi}{\lambda}\left[\sqrt{f_{1}^{2}+\left(x-x_{1}\right)^{2}+\left(y-y_{1}\right)^{2}}-f_{1}\right]+\frac{\pi}{6}$,

where $x_{1}=-75 \mu \mathrm{m}, y_{1}=75 \mu \mathrm{m}, f_{1}=4.8 \mathrm{~mm}$, and $\left(x_{1}, y_{1}, f_{1}\right)$ represents the focal point of the transmitted wave. Meanwhile, to obtain the polarization multiplexing metasurface [see Fig. 4(b)] we designed, the meta-atoms B, whose phase distribution satisfies Eq. (9) by $75 \mu \mathrm{m}$ along the positive $x$ direction and $75 \mu \mathrm{m}$ along the negative $y$ direction, are translated. It is worth noting that the use of such a spatial interleaving method needs to ensure that there is no overlap between the meta-atoms A and B. Figures 4(c) and 4(d), respectively, show the intensity distribution of the two EP components on the $x z$ plane under RCP incidence, implying that the outgoing wave generated by the metasurface will be focused on a certain point. In order to further describe the characteristics of the outgoing wave, Fig. 4(e) depicts the intensity profile of the corresponding outgoing wave on the focal plane. It can be seen that the EP waves generated by the meta-atoms A and B are superimposed on the focal plane to generate $x$-LP waves.

Next, we discuss the transmission results of the metasurface under LCP, $x$-LP, and $y$-LP incidences. Figure 5(a) exhibits the
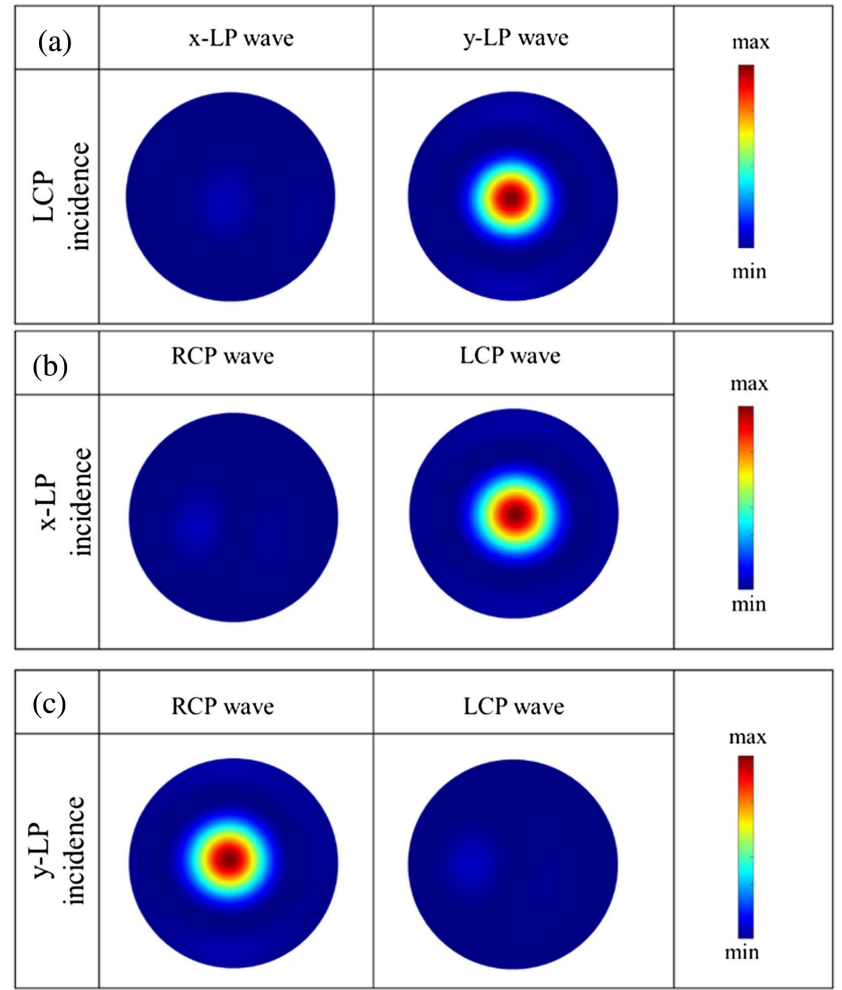

Fig. 5. (a)-(c) Intensity profiles of the desired polarization and the corresponding orthogonal polarization in the cases of $L C P, x-L P$, and $y$-LP incidences. 
intensity distributions of the expected polarization and corresponding orthogonal polarization on the focal plane when the incident wave is LCP. The simulated results indicate that the metasurface composed of meta-atoms $\mathrm{A}$ and $\mathrm{B}$ can generate focused $y-\mathrm{LP}$ waves under LCP incidence. Figures 5(b) and 5(c) illustrate the simulated results of $\mathrm{CP}$ waves under $x-\mathrm{LP}$ and $y-\mathrm{LP}$ waves, respectively. In this part, the proposed metasurface with polarization multiplexing can generate CP (LP) waves under LP (CP) incidences, proving the feasibility of such design.

\section{Conclusions}

We present an all-silicon metasurface composed of meta-atoms A with the phase difference of $2 \pi / 3$ along the $x$ and $y$ directions to generate specific EP waves under different polarized waves. For instance, when an RCP wave is incident, the metasurface will generate LCP and RCP waves with the same phase but different amplitudes, and then they are superimposed to engender EP waves. Furthermore, we introduce meta-atoms $B$ with a phase difference of $\pi / 3$ and meta-atoms $A$ to construct a metasurface that can realize bidirectional polarization conversion between linear polarization and circular polarization. The simulated results are in good agreement with the deduced results, verifying the good performance of our design concepts. We envisage that such an approach may spur a new wave of photonics devices and can effectively extend the polarization multiplexing metasurfaces to a broader range.

\section{Acknowledgement}

This work was supported by the National Natural Science Foundation of China (Nos. 61675147, 61735010, and 91838301) and National Key Research and Development Program of China (No. 2017YFA0700202).

\section{References}

1. C. Pfeiffer and A. Grbic, "Metamaterial Huygens' surfaces: tailoring wave fronts with reflectionless sheets," Phys. Rev. Lett. 110, 197401 (2013).

2. F. Walter, G. X. Li, C. Meier, S. Zhang, and T. Zentgraf, "Ultrathin nonlinear metasurface for optical image encoding," Nano Lett. 17, 3171 (2017).

3. N. F. Yu and F. Capasso, "Flat optics with designer metasurfaces," Nat. Mater. 13, 139 (2014).

4. S. Q. Chen, Z. Li, Y. B. Zhang, H. Cheng, and J. G. Tian, "Phase manipulation of electromagnetic waves with metasurfaces and its applications in nanophotonics," Adv. Opt. Mater. 6, 1800104 (2018).

5. M. Q. Mehmood, S. T. Mei, S. Hussain, K. Huang, S. Y. Siew, L. Zhang, T. H. Zhang, X. H. Ling, H. Liu, J. H. Teng, A. Danner, S. Zhang, and C. W. Qiu, "Visible-frequency metasurface for structuring and spatially multiplexing optical vortices," Adv. Mater. 28, 2533 (2016).

6. W. W. Liu, S. Q. Chen, Z. C. Li, H. Cheng, P. Yu, J. X. Li, and J. G. Tian, "Realization of broadband cross-polarization conversion in transmission mode in the terahertz region using a single-layer metasurface," Opt. Lett. 40, 3185 (2015).
7. Q. L. Yang, X. Y. Chen, Q. Xu, C. X. Tian, Y. H. Xu, L. Q. Cong, X. Q. Zhang, Y. F. Li, C. H. Zhang, X. X. Zhang, J. G. Han, and W. L. Zhang, "Broadband terahertz rotator with an all-dielectric metasurface," Photonics Res. 6, 1056 (2018).

8. Z. L. Deng, Q. A. Tu, Y. J. Wang, Z. Q. Wang, T. Shi, Z. W. Feng, X. C. Qiao, G. P. Wang, S. M. Xiao, and X. P. Li, "Vectorial compound metapixels for arbitrary nonorthogonal polarization steganography," Adv. Mater. 33, 2103472 (2021).

9. W. W. Wan, J. Gao, and X. D. Yang, "Full-color plasmonic metasurface holograms," ACS Nano 10, 10671 (2016).

10. L. Wang, S. Kruk, H. Z. Tang, T. Li, I. Kravchenko, D. N. Neshev, and Y. S. Kivshar, "Grayscale transparent metasurface holograms," Optica 3, 1504 (2016).

11. E. Maguid, I. Yulevich, D. Veksler, V. Kleiner, M. L. Brongersma, and E. Hasman, "Photonic spin-controlled multifunctional shared-aperture antenna array," Science 352, 1202 (2016).

12. Y. Xu, Q. Li, X. Zhang, M. Wei, Q. Xu, Q. Wang, H. Zhang, W. Zhang, C. Hu, Z. Zhang, C. Zhang, X. Zhang, J. Han, and W. Zhang, "Spin-decoupled multifunctional metasurface for asymmetric polarization generation," ACS Photonics 6, 2933 (2019).

13. F. Aieta, M. A. Kats, P. Genevet, and F. Capasso, "Multiwavelength achromatic metasurfaces by dispersive phase compensation," Science 347, 1342 (2015).

14. S. M. Wang, P. C. Wu, V. C. Su, Y. C. Lai, M. K. Chen, H. Y. Kuo, B. H. Chen, Y. H. Chen, T. T. Huang, J. H. Wang, R. M. Lin, C. H. Kuan, T. Li, Z. L. Wang, S. N. Zhu, and D. P. Tsai, "A broadband achromatic metalens in the visible," Nat. Nanotechnol. 13, 227 (2018).

15. K. Huang, H. Liu, S. Restuccia, M. Q. Mehmood, S. T. Mei, D. Giovannini, A. Danner, M. J. Padgett, J. H. Teng, and C. W. Qiu, "Spiniform phaseencoded metagratings entangling arbitrary rational-order orbital angular momentum," Light Sci. Appl. 7, 17156 (2018).

16. Z. Yue, J. Y. Liu, J. T. Li, J. Li, C. L. Zheng, G. C. Wang, M. Y. Chen, H. Xu, Q. Wang, X. H. Xing, Y. T. Zhang, Y. Zhang, and J. Q. Yao, "Multifunctional terahertz metasurfaces for polarization transformation and wavefront manipulation," Nanoscale 13, 14490 (2021).

17. J. W. Zeng, L. Li, X. D. Yang, and J. Gao, "Generating and separating twisted light by gradient-rotation split-ring antenna metasurfaces," Nano Lett. 16, 3101 (2016).

18. C. L. Zheng, G. C. Wang, J. Li, J. T. Li, S. L. Wang, H. L. Zhao, M. Y. Li, Z. Yue, Y. T. Zhang, Y. Zhang, and J. Q. Yao, "All-dielectric metasurface for manipulating the superpositions of orbital angular momentum via spin-decoupling," Adv. Opt. Mater. 9, 2002007 (2021).

19. X. M. Ding, F. Monticone, K. Zhang, L. Zhang, D. L. Gao, S. N. Burokur, A. de Lustrac, Q. Wu, C. W. Qiu, and A. Alu, "Ultrathin Pancharatnam-Berry metasurface with maximal cross-polarization efficiency," Adv. Mater. 27, 1195 (2015).

20. P. C. Wu, W. Y. Tsai, W. T. Chen, Y. W. Huang, T. Y. Chen, J. W. Chen, C. Y. Liao, C. H. Chu, G. Sun, and D. P. Tsai, "Versatile polarization generation with an aluminum plasmonic metasurface," Nano Lett. 17, 445 (2017).

21. J. P. B. Mueller, N. A. Rubin, R. C. Devlin, B. Groever, and F. Capasso, "Metasurface polarization optics: independent phase control of arbitrary orthogonal states of polarization," Phys. Rev. Lett. 118, 113901 (2017).

22. Y. Xu, H. Zhang, Q. Li, X. Zhang, Q. Xu, W. Zhang, C. Hu, X. Zhang, J. Han, and W. Zhang, "Generation of terahertz vector beams using dielectric metasurfaces via spin-decoupled phase control," Nanophotonics 9, 3393 (2020).

23. Y. Y. Yuan, S. Sun, Y. Chen, K. Zhang, X. M. Ding, B. Ratni, Q. Wu, S. N. Burokur, and C. W. Qiu, "A fully phase-modulated metasurface as an energy-controllable circular polarization router," Adv. Sci. 7, 2001437 (2020).

24. Y. Y. Yuan, K. Zhang, B. Ratni, Q. H. Song, X. M. Ding, Q. Wu, S. N. Burokur, and P. Genevet, "Independent phase modulation for quadruplex polarization channels enabled by chirality-assisted geometric-phase metasurfaces," Nat. Commun. 11, 4186 (2020).

25. C. L. Zheng, J. Li, G. C. Wang, S. L. Wang, J. T. Li, H. L. Zhao, H. P. Zang, Y. Zhang, Y. T. Zhang, and J. Q. Yao, "Fine manipulation of terahertz waves via all-silicon metasurfaces with an independent amplitude and phase," Nanoscale 13, 5809 (2021). 